\title{
Supernovae from yellow, blue supergiants: origin and consequences for stellar evolution
}

\author{
G. Meynet ${ }^{1}$, J. Groh ${ }^{1}$, C. Georgy ${ }^{2}$, H. Saio ${ }^{3}$ and R. Kudritzki ${ }^{4}$ \\ ${ }^{1}$ Geneva Observatory, University of Geneva, Maillettes 51, 1290, Sauverny, Switzerland \\ email: georges.meynet@unige.ch \\ ${ }^{2}$ Astrophysics, Lennard-Jones Laboratories, EPSAM, Keele University, Staffordshire, ST5 \\ $5 \mathrm{BG}, \mathrm{UK}$ \\ ${ }^{3}$ Astronomical Institute, Graduate School of Science, Tohoku University, Sendai, Japan \\ ${ }^{4}$ Institute for Astronomy, University of Hawaii, 2680 Woodlawn Drive, Honolulu, HI, 96822, \\ USA
}

\begin{abstract}
A few core collapse supernovae progenitors have been found to be yellow or blue supergiants. Weshall discuss possible scenarios involving single and close binary evolution allowing to explain this kind of corecollapse supernova progenitors. According to stellar models for both single and close binaries, blue supergiants, at theend of their nuclear lifetimes and thus progenitors of core collapse supernovae, present very different characteristicsfor what concerns their surface compositions, rotational surface velocities and pulsational properties with respect toblue supergiants in their core helium burning phase. We discuss how the small observed scatter of the flux-weightedgravity-luminosity (FWGL) relation of blue supergiants constrains the evolution of massive stars after the Main-Sequence phase and the nature of the progenitors of supernovae in the mass range between 12 and 40 solarmasses. The present day observed surface abundances of blue supergiants, of their pulsational properties, as well asthe small scatter of the FWGL relation provide strong constraints on both internal mixing and mass loss in massivestars and therefore on the end point of their evolution.
\end{abstract}

